\title{
Fabrication of crystalline submicro-to-nano carbon wire for achieving high current density and ultrastable current
}

\author{
Jufeng Deng ${ }^{1,2}$, Chong Liu ${ }^{1 凶}$, Dian song ${ }^{3}$ and Marc Madou $2,3,4 凶$
}

\begin{abstract}
Crystalline carbon nanowire arrays were fabricated taking advantage of near-field electrospinning and stress decyanation. A novel fabrication method for carbon nanowires with radii ranging from $\sim 2.15 \mu \mathrm{m}$ down to $\sim 25 \mathrm{~nm}$ was developed based on implementing nitrogen pretreatment on the silica surface and then aligning polymer nanofibers during near-field electrospinning at an ultralow voltage. Stress decyanation was implemented by subsequently pyrolyzing a polymer nanofiber array on the silica surface at $1000^{\circ} \mathrm{C}$ for $1 \mathrm{~h}$ in an $\mathrm{N}_{2}$ atmosphere, thus obtaining a crystalline carbon nanowire array with a nanostructured surface. Various crystalline nanostructures were fabricated on the nanowire surface, and their electrochemical performance was evaluated by cyclic voltammetry (CV) and electrochemical impedance spectroscopy (EIS). Crystalline carbon wires with diameters ranging from micrometers to submicrometers displayed carbon nanoelectrode-like behavior with their CV curve having a sigmoidal shape. A highly crystalline carbon nanowire array showed distinct behavior, having a monotonically increasing straight line as its CV curve and a semicircular EIS spectrum; these results demonstrated its ultrastable current, as determined by electron transfer. Furthermore, nanocrystalline-structured carbon wires with diameters of $\sim 305 \mathrm{~nm}$ displayed at least a fourfold higher peak current density during CV $\left(4000 \mathrm{~mA} / \mathrm{m}^{2}\right)$ than highly crystalline carbon nanowires with diameters of $\sim 100 \mathrm{~nm}$ and porous microwires with diameters of $\sim 4.3 \mu \mathrm{m}$.
\end{abstract}

\section{Introduction}

The attractive merit of carbon wires derives from carbon microstructures integrating high-performance with abundant functionalities ${ }^{1,2}$. There are several available microstructures of carbon materials, such as glassy carbon, diamond, and graphite. Carbon nanowires with glassy carbon structures have been used in many different applications, such as in high-power supercapacitors ${ }^{3}$, electrochemical biosensors ${ }^{4}$ and high-energy rechargeable batteries $^{5}$. To upgrade all of the intrinsic properties in the

\footnotetext{
Correspondence: Chong Liu (chongl@dlut.edu.cn) or

Marc Madou (mmadou@uci.edu)

${ }^{1}$ School of Mechanical Engineering, Dalian University of Technology, Dalian 116023, China

${ }^{2}$ Mechanical and Aerospace Engineering, University of California, Irvine, CA 92617, USA

Full list of author information is available at the end of the article
}

case of conventional carbon nanowires with glassy carbon structures, the principal philosophy emphasizes the interconnected graphitic structure, which provides an extraordinary combination of mechanical, electrical, and thermal properties (strength ${ }^{6}$ up to $20 \mathrm{GPa}$, electrical conductivity $^{6}$ of $1.5 \times 10^{6} \mathrm{~S} / \mathrm{m}$ and thermal conductivity ${ }^{7}$ of $5000 \mathrm{~W} / \mathrm{m} \cdot \mathrm{K})$. Compared to glassy carbon nanowire configurations, graphitized carbon nanowires offer several advantages, such as their extraordinary mechanical properties, which includes their elastic modulus ${ }^{8}$ $(1.1 \mathrm{TPa})$, tensile $\operatorname{strength}^{8}(\sim 130 \mathrm{GPa})$ and notable flexibility, and their excellent electron transport performance $^{9}$, which includes their extremely high electric conductivity $\left(\sim 10^{8} \mathrm{~S} / \mathrm{m}\right)$, carrier mobility $\left(2000000 \mathrm{~cm}^{2} /\right.$ $\mathrm{V} \cdot \mathrm{s})$ and ampacity $\left(1-2 \mathrm{GA} / \mathrm{cm}^{2}\right)$. These advantages demonstrate the abundant functionalities of graphitized

\section{(c) The Author(s) 2022}

(c) (i) Open Access This article is licensed under a Creative Commons Attribution 4.0 International License, which permits use, sharing, adaptation, distribution and reproduction c. in any medium or format, as long as you give appropriate credit to the original author(s) and the source, provide a link to the Creative Commons license, and indicate if changes were made. The images or other third party material in this article are included in the article's Creative Commons license, unless indicated otherwise in a credit line to the material. If material is not included in the article's Creative Commons license and your intended use is not permitted by statutory regulation or exceeds the permitted use, you will need to obtain permission directly from the copyright holder. To view a copy of this license, visit http://creativecommons.org/licenses/by/4.0/. 
carbon nanowires for use in various applications, such as in electrical conductors ${ }^{10}$, supercapacitors ${ }^{11}$, actuators, and solar devices ${ }^{12}$, showing more applicability than conventional carbon nanowires. Regarding electrochemical energy storage, graphitized carbon nanowires are promising for enhancing energy and power densities.

The thermal reduction and high-temperature annealing of graphite oxide wire made by a wet-spinning process have been proposed to produce highly graphitized carbon wires but are limited to micron-scale fabrication $^{13}$. For the electrochemical applications mentioned above, the performance is typically improved by increasing the surface area of graphitized carbon nanowires, which can be achieved by decreasing the diameter of graphitized carbon nanowires. Most of the techniques that have been proposed for fabricating graphitized nanoscale carbon wires include mechanical stress pyrolysis ${ }^{14}$ and chemical vapor deposition ${ }^{15}$. The doping approaches involved in these techniques cause additional substances to be added into the carbon wires, which affects the electrochemical behavior of the carbon wire. This has led to extensive research into the pyrolysis of near-field electrospun fibers (PNFEFs), which enables advanced applications in electrochemical sensing, energy storage, and stem cells.

PNFEFs are dedicated to manufacturing new classes of well-defined carbon nanostructures. In this process, polymer nanofibers are arrayed by the near-field electrospinning of a precursor solution and subsequently converted into pyrolytic carbon nanowires through high-temperature treatment $\left(>800^{\circ} \mathrm{C}\right)$ in an inert atmosphere. This method offers excellent control of feature sizes, density per unit area, and the capability to pattern carbon nanowires. By introducing carbon scaffolds into this process, a dramatic decrease in the fiber diameter leads to ultrathin carbon nanowires $(\sim 5 \mathrm{~nm})^{16}$. This technique is mostly established because the highthroughput fabrication of carbon nanowires can be easily achieved.

Recently, highly graphitized carbon nanowires ${ }^{17}$ have been developed on carbon scaffolds using near-field electrospinning, pyrolysis, electrodeposition, and chemical vapor deposition. Clearly, these techniques are still inseparable from the participation of nickel catalysts, resulting in an inability to remove the nickel from the carbon wire. Thus, to date, the electrochemical behavior of highly crystalline carbon nanowires with graphitic structures have not been improved. Here, we present the fabrication and characterization of highly crystalline carbon nanowire (HCCN) arrays with well-controlled wireto-wire spacing. For this purpose, a novel method for the fabrication of highly crystalline carbon nanowires is implemented, permitting control of the wire diameter, wire-to-wire spacing, and degree of graphitization. By the programmed movement of a linear stage during near-field electrospinning (NFES), the polymer nanofibers are arrayed on a silicon substrate coated with silica $(500 \mathrm{~nm}$ thickness). Before depositing the polymer nanofiber arrays, the silica surface deposited by polymer fibers is pretreated at $1000{ }^{\circ} \mathrm{C}$ in a nitrogen atmosphere. To convert the polymer fibers into carbon nanowires, a silicon chip with carbon dioxide and polymer fibers is pyrolyzed at $1000{ }^{\circ} \mathrm{C}$ in a nitrogen atmosphere. The microstructure of the carbon wires is evaluated by atomic force microscopy and Raman spectroscopy. Additionally, the fabricated HCCNs are characterized electrochemically with cyclic voltammetry and electrochemical impedance spectroscopy (ESI).

\section{Results and discussion}

\section{Fabrication of polymer nanofiber arrays using near-field electrospinning (NFES)}

To attain the thinnest polymer fibers at the lowest applied voltages in NFES, one important procedure concerns the protocol for preparing the ink by the dissolution of polyacrylonitrile (PAN) in $N, N$-dimethylformamide (DMF). A heat treatment from 60 to $126^{\circ} \mathrm{C}$ is found to be accompanied by a conductivity change, which indicates the highest conductivity at $106^{\circ} \mathrm{C}^{16}$. The conductivity data can be collected by a conductivity meter (OAKTON CON 510 Series) with the ability to show both conductivity and solution temperature. The conductivity plays a role in inducing sufficiently large electrical stress, which makes it possible to initiate a nano jet during near-field electrospinning. With a drumto-needle distance of $5 \mathrm{~mm}$, the polymer jet does not initiate even at $1500 \mathrm{~V}$ because the electrostatic force cannot overcome the surface tension at the droplet-air interface. However, touching the ink droplet at the ejector needle tip with the rotating drum causes the electrical stress to become large enough to counterbalance the surface tension stress, giving rise to the formation of a Taylor cone and jet initiation at a voltage as low as $500 \mathrm{~V}$ (see Fig. 1a). When using a PAN ink heated to either $60^{\circ} \mathrm{C}$ or $126^{\circ} \mathrm{C}$, jet initiation at $500 \mathrm{~V}$ is not achieved by the same touching procedure, which demonstrates the key role of conductivity for initiating the jet during NFES.

A porous absorbent paper mounted around the base of the ejector needle thins the liquid layer around the needle at low feed rates $(1 \mathrm{~nL} / \mathrm{min})$. The resulting electric field intensity at the liquid-air interface is substantially higher than that of the nozzle without droplet shaping at the same voltage ${ }^{18}$. This in turn results in the decrease in the applied voltage from 500 to $35 \mathrm{~V}$, which is far below any current low-voltage NFES practice (see Fig. 1a). During NFES, continuous jetting at an ultralow applied voltage of $35 \mathrm{~V}$ becomes possible (see Fig. 1a). This reduction in the 


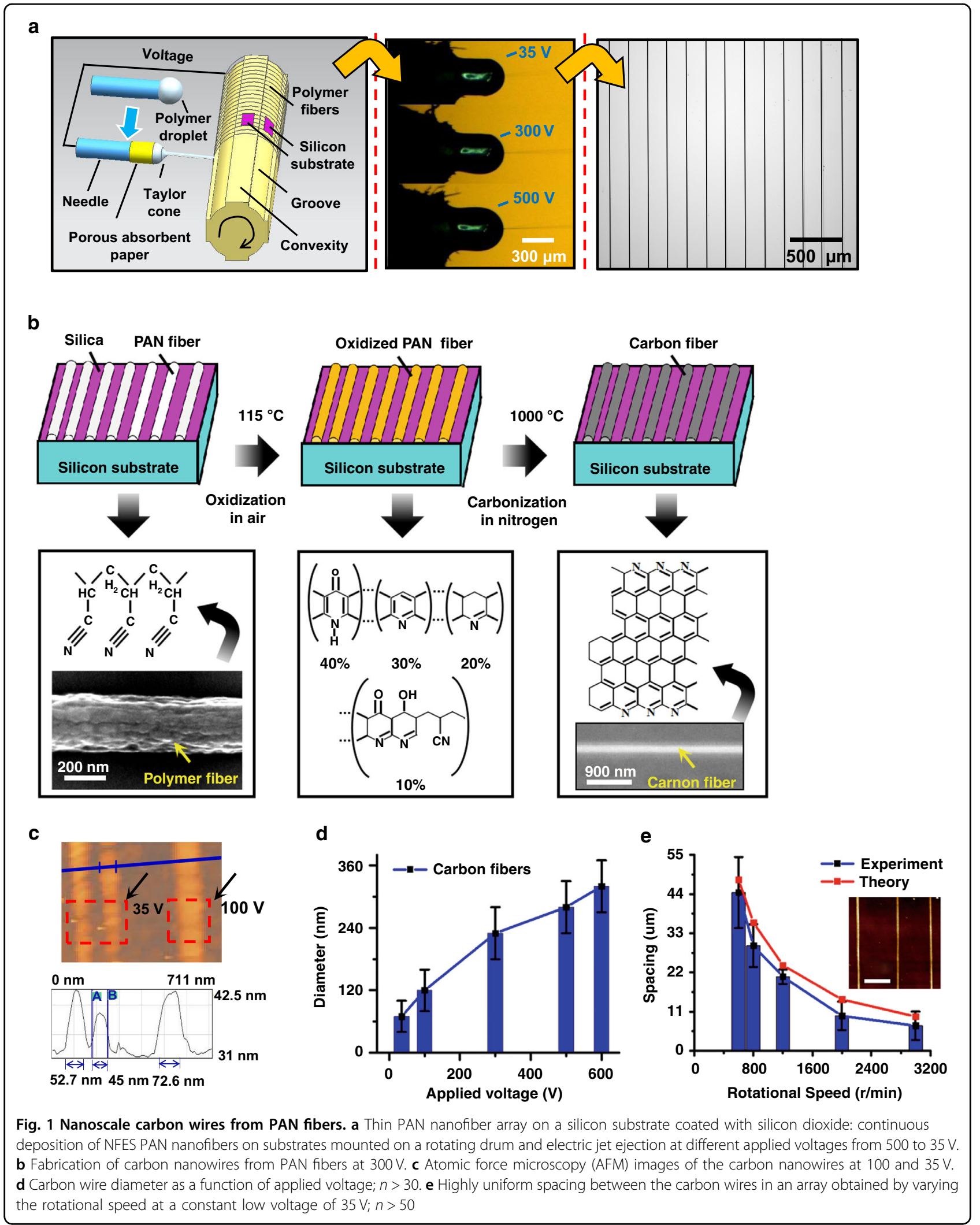


working voltage causes a decrease in the jet diameter, as described by Eq. (1): ${ }^{16}$ movement of the stage, polymer nanofibers with diameters as low as $50 \mathrm{~nm}$ diameter are aligned and depos-

$$
h=\text { InverseFunction }\left\{\left[-\frac{E^{2} K^{2} \operatorname{Inx}}{8 I^{3}}+\frac{E^{2} K^{2} \operatorname{In}\left(-2 I+E K x^{2}\right)}{16 I^{3}}+\frac{1}{8 I x^{4}}+\frac{E K}{8 I^{2} x^{2}}\right]\left[\frac{E x}{2 Q^{3} \sqrt{\beta}}+C_{1}\right]\right\}
$$

where $h$ is the cross-sectional fiber radius, $E$ is the electric field strength, $K$ is the electric conductivity, $I$ is the total current of the electrified jet, $x$ is the distance between the needle and drum along the axis of the needle, $Q$ is the volume flow rate, $\beta=\epsilon^{3 / 2}-1$ is the dimensionless conductivity of the fluid ${ }^{19}, \epsilon$ is the dielectric constant and $C_{1}$ is a constant.

After the polymer jet is initiated at various voltages from 500 to $35 \mathrm{~V}$, the alignment of polymer nanofibers on the silicon substrate is implemented by the programmed movement of a linear stage on which the needle is affixed (see Fig. 1a). In this automated positioning system, the rotational speed $\omega$ of a drum and the linear speed $v$ of the nozzle moving along the surface of that drum can readily tune the fiber-to-fiber spacing $d$ as follows:

$$
d=v / \omega
$$

where $v$ is the linear speed of the nozzle moving along the surface of the drum, and $\omega$ is the rotational speed of the drum. In comparison with traditional electrospinning, shear force spinning during NFES affords a more uniform fiber-to-fiber spacing, as indicated by an $\sim 2$-fold decrease in the relative standard deviation of the achieved spacings ${ }^{19}$. Therefore, a rotating drum is introduced to add shear force into our electrospinning system, ultimately resulting in the more uniform alignment of polymer fibers shown in Fig. 1a. A comparison between the theoretical and experimental results is illustrated in Fig. 1e, revealing that maximizing the rotational speed $(\omega)$ and minimizing the linear stage speed $(v)$ makes it possible to lower the fiber spacing to as small as $\sim 2.5 \mu \mathrm{m}$; thus, the density per unit area of the carbon nanowires on the silicon substrate can be maximized. Controlling the wire-to-wire spacing and carbon nanowire diameter makes it possible to obtain materials with higher sensitivity and stability for biosensing applications and kinetic studies ${ }^{20}$.

At a constant voltage of $35 \mathrm{~V}$, polymer fibers in the convex parts of the drum are thinner than those in the grooved parts (see the right image in Fig. 1a). This is most likely due to the increase in the mechanical stretching of the nanofibers between the point of contact on the silicon substrate and droplet. By implementing a very low NFES working voltage of $35 \mathrm{~V}$ and programming the linear ited on silicon substrates fixed in the convex areas of the drum. The limitations of the proposed method lie in the dependency of initiating the jet and arraying fibers on the rotating drum. Instead of arraying fibers, making three-dimensional fiber scaffolds or films with this fabrication method has to undergo breaks in the lines due to the instability of the fluid flow inside the needle during long-term jetting.

Pyrolysis at $1000^{\circ} \mathrm{C}$ leads to radial and longitudinal contraction as well as an enhanced tensile strength of the stabilized PAN nanofibers because of the increased carbon content ${ }^{21-23}$. As a result, a radial shrinkage of less than $15 \%$ in Fig. $1 \mathrm{~b}$ is observed, which is lower than the 47.7 to $90 \%$ shrinkage ${ }^{17}$ from freely suspended polymeric nanofibers. This is most likely due to the strong attachment of the polymeric nanofiber to the silicon substrate, which derives from the impact of the polymer fiber on the silicon substrate during deposition. A combination of the effects of surface wetting and thermal expansion coefficient mismatch at the interface contributes to the possible interaction between polymeric nanofibers and a silicon substrate. The height of the carbon wire is much smaller than the width in Fig. 1c, showing fiber deformation caused by strong attachment. Due to lower shrinkage, the fabrication of a thin carbon wire largely depends on reducing the diameter of the polymer fiber. Ultralowvoltage (35 V) NFES allows for the smallest polymer fiber diameter, achieving thin carbon nanowires with diameters down to $45 \mathrm{~nm}$, as shown in Fig. 1c. The effect of the applied voltage on the thickness of the carbon nanowires in Fig. $1 \mathrm{~d}$ clearly reveals a decrease in the average diameter of the carbon nanowires with decreasing applied voltage during NFES. Since we initiate jetting with an ultralow voltage $(35 \mathrm{~V})$, the resulting carbon nanowires have an average diameter of 60 nanometers, which is far thinner than typical carbon nanowire diameters ${ }^{24}$. The dependency of the electrical properties on the size demonstrates that thinner carbon nanowires tend to far exceed the electrical properties of traditional carbon fibers $^{25}$.

\section{Effect of aligning carbon nanowires and implementing a nitrogen pretreatment on the nanocrystalline structuring of carbon nanowires}

Many reports conclude that the degree of a graphitic microstructure in carbon wires is related to the physical 
synthesis conditions, such as stress-induced routes ${ }^{14}$, torque-applied stretching ${ }^{26}$, and mechanical and electrostatic stretching ${ }^{27}$. A novel method for improving the graphitic microstructure of carbon wires on silica surfaces is developed by structuring nanocrystalline materials in this paper, which lays a foundation for studying the electrochemical properties of graphitic microstructures. To understand the mechanism of structuring nanocrystalline materials, disorderly carbon nanowires and partially aligned carbon wires are introduced as reference objects.
After jet initiation at $500 \mathrm{~V}$ in Fig. 1a, an increase in the applied voltage to $1200 \mathrm{~V}$ allows for the continuous electrospinning of a single, stable filament from a droplet at a flow rate of $1 \mathrm{nl} / \mathrm{min}$. In this case, increasing the needle-drum distance from $1.85 \mathrm{~mm}$ to $10 \mathrm{~mm}$ during NFES results in the formation of disorderly polymer fibers and partially aligned polymer fibers onto the rotating drum. Subsequent stabilization in the air at $115^{\circ} \mathrm{C}$ and then carbonization in a nitrogen atmosphere at $1000^{\circ} \mathrm{C}$ (at a heating rate of $15^{\circ} \mathrm{C} / \mathrm{min}$ from 260 to $1000^{\circ} \mathrm{C}$ ) transform such polymer fibers into carbon nanowires, as

a

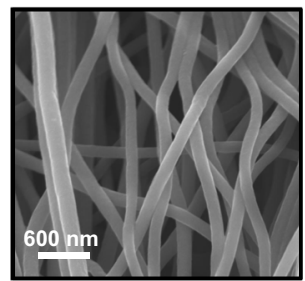

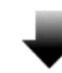
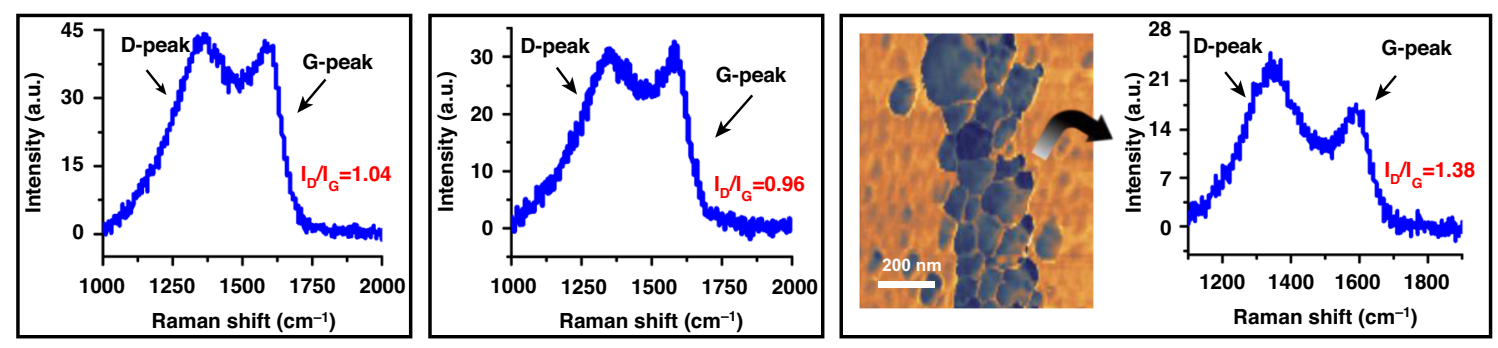

b
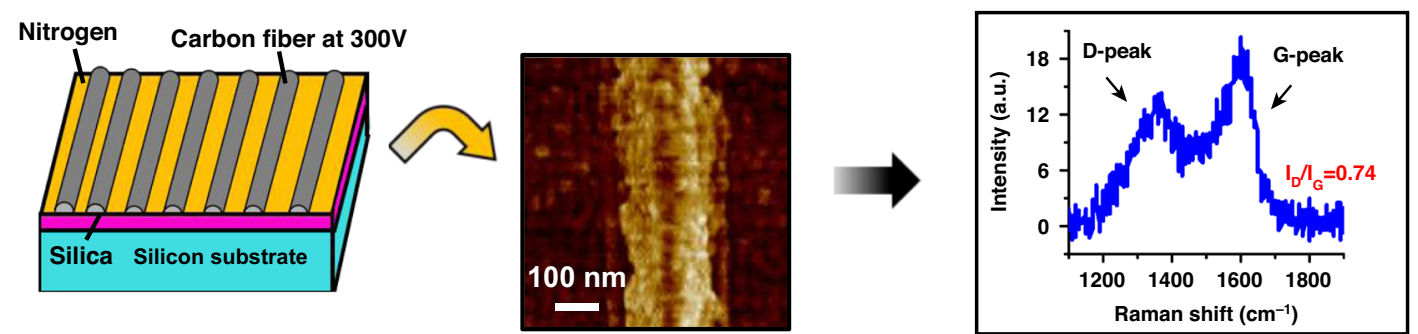

C
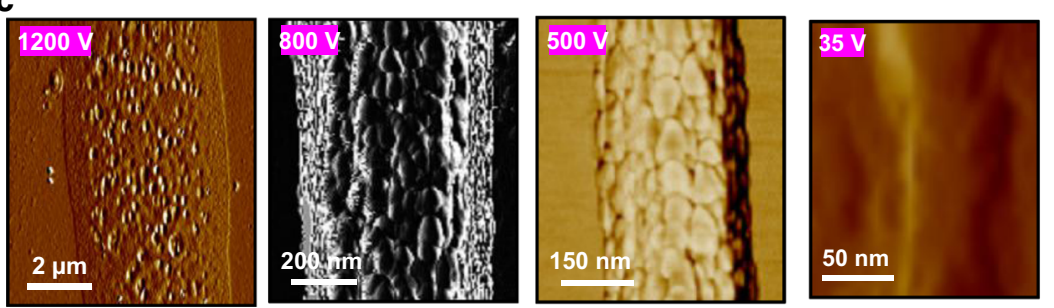

Fig. 2 Effect of voltage-dependent nanowire size on the crystalline structure in regard to nitrogen pretreatment. a Limiting the radial and axial shrinkage to develop tensile stress. b Crystalline carbon nanowires on silica surfaces processed with nitrogen pretreatment at $300 \mathrm{~V}$. c AFM phase images of carbon nanowires at 1200,800, 500, and $35 \mathrm{~V}$. d Transformation of the crystalline structure from porous to highly crystalline; $n>35$ 
shown in Fig. 2a. In general, the graphitic nature of PANderived carbons is evaluated based on Raman spectroscopy, a standard nondestructive analysis tool. As shown in Fig. 2a, we observe the $D$ and $G$ peaks centered at approximately 1350 and $1590 \mathrm{~cm}^{-1}$, respectively. The spectrum of pure graphite shows a strong $G$-peak due to the in-plane bond-stretching motion of pairs of $C s p^{2}$-bonded atoms, while the $D$-peak is more pronounced in the presence of defects such as bond-angle disorder, bond-length disorder, vacancies, and edge defects ${ }^{28}$. The intensity ratio of the $D$ and G-peaks $\left(I_{\mathrm{D}} / I_{\mathrm{G}}\right)$ is proportional to the in-plane correlation length and corresponds to the amount of disorder present in the carbon wire ${ }^{29}$. The lower the value of $I_{\mathrm{D}} / I_{\mathrm{G}}$ is, the higher the crystalline microstructure or the lower the disordered (amorphous) nature of the carbon.

Because of the disordered arrangement of carbon wires (Fig. 2a), the tensile force during the carbonization process does not effectively stretch the fibers along the longitudinal direction, resulting in a high-intensity ratio of 1.04 (Fig. 2a). After aligning the carbon wires along the longitudinal direction (Fig. 2a), the resulting intensity ratio of 0.96 (Fig. 2a) is slightly lower than 1.04, showing a higher crystallinity or a lower amorphous nature. Although the alignment of fibers demonstrates the possibility of developing tension to improve crystallinity, the microstructure of this carbon is still glassy in nature based on the results (Fig. 2a).

The formation of glassy carbon during pyrolysis derives from the curved structures of the penta- and heptacarbon rings ${ }^{14}$. To implement this alignment process for curved structures, a mechanical treatment that changes axial stress from compressive stress to tensile stress is applied to the PAN fibers for a remarkable increase in the graphitic structure of the resulting carbons. Here, the deformation introduced by aligning the polymer nanofibers on a silica surface (Fig. 2a) plays a somewhat similar role to that of mechanical treatment, as described by Maziar et al. ${ }^{14}$. The resulting strong attachment of the polymeric nanofiber to the silicon substrate limits radial shrinkage during stabilization and carbonization, thus forming tension stress in the axial and radial directions. Upon increasing the rotational speed of the drum, the bond of the nanofiber to the silica surface becomes strong enough to counterbalance the tension developed during pyrolysis. Atomic force microscopy allows for a detailed study of the resulting carbon wire microstructure. Visual examination of the atomic-resolution micrographs illustrates the evolution of the developed carbon wire microstructures. Since these two forces have opposite effects on the radial shrinkage of fibers, polymer fibers are transformed into nanograin-based carbon wires (AFM phase images on the left side of Fig. 2a). The resulting intensity ratio of 1.38 is much higher than 0.96 and 1.04, thus poorly crystalline carbon wires are formed.
Nitrogen pretreatment is performed by placing the silicon chip with the carbon dioxide surface (left side of Fig. 2b) in a quartz glass tube filled with nitrogen for heat treatment at $1000^{\circ} \mathrm{C}$ to dramatically overcome the breaking of fiber into nanograins during pyrolysis. Once the polymer fibers are deposited on the silica surface pretreated with nitrogen, carbonization transforms the polymer fiber structure into a defect-free carbon wire (AFM phase images in the middle of Fig. 2b). The transition from a nanograin-based microstructure to a defect-free microstructure is accompanied by a decrease in the $I_{\mathrm{D}} / I_{\mathrm{G}}$ ratio from 1.38 to 0.74 (Fig. $2 \mathrm{a}, \mathrm{b}$ ), thus forming highly crystalline carbon wires. A previous study showed that the graphitic structure in carbon wires showed a remarkable increase with a decrease in the $I_{\mathrm{D}} / I_{\mathrm{G}}$ ratio from 1.26 to $0.69^{14}$. The highly crystalline carbon wires are believed to have a much more graphitic structure.

\section{Effect of nanowire diameter on crystalline structuring}

From Fig. 2c, d, it is clear that stress management is crucial to yield highly crystalline carbon nanowires. The tensile stress on the cross-section of the fibers becomes more pronounced with decreasing diameters of the fibers, so we also exploited thinning the wires to further improve the graphitic structure. The effect of the nanowire diameter, which is dependent on the applied voltage in Fig. 1d, on the microstructure and crystallinity of the carbon nanowires on the silica surface pretreated with nitrogen is illustrated in Fig. 2c, d. Further investigation into the decrease in wire diameter from $\sim 5.05 \mu \mathrm{m}$ to $\sim 45 \mathrm{~nm}$ (Fig. 2c, d) reveals an evolution from a porous microstructure to a highly crystalline nanostructure via nanocrystalline structuring. A Raman spectrometer with a DXR microscope and equipped with a $532 \mathrm{~nm}$ excitation laser was selected to evaluate the crystallinity of the microstructures in the carbon nanowires fabricated at 1200,500 , and $35 \mathrm{~V}$. The intensity ratio of 0.78 in Fig. 2d is much lower than that of 1.41 in Fig. 2d, corresponding to the higher degree of crystallinity of the nanocrystalline microstructure. This improvement in crystallinity may be attributed to the increase in tensile stress in the axial direction due to the decrease in diameter from $\sim 5.05 \mu \mathrm{m}$ to $\sim 305 \mathrm{~nm}$ (Fig. 2c). Upon further reduction of the carbon nanowire diameter from $\sim 305 \mathrm{~nm}$ to $\sim 45 \mathrm{~nm}$ (Fig. 2c), the microstructure is transformed into an even more crystalline microstructure, which is gleaned from the decrease in $I_{\mathrm{D}} / I_{\mathrm{G}}$ from 0.78 to 0.64 (Fig. $2 \mathrm{~d}$ ). Figure $2 \mathrm{~d}$ illustrates the correlation between the crystallinity of the microstructures and the applied voltage. Clearly, the polymer fibers obtained at the lowest voltages result-after pyrolysis-in the highly crystalline carbon nanowires owing to their highly graphitized structures. 


\section{Effect of decyanation on crystalline structuring}

The effect of tension stress on crystalline structuring is accompanied by decyanation reactions (named stress decyanation). A previous report showed that relying on denitrogenation and decyanation reactions to remove impurity elements during carbonization (Fig. 3a) resulted in the formation of carbon sheet-like layers from carbonlike ribbons ${ }^{2}$. To correlate the influence of the denitrogenation and decyanation reactions with the graphitic structure, Raman spectroscopy, and X-ray photoelectron

a
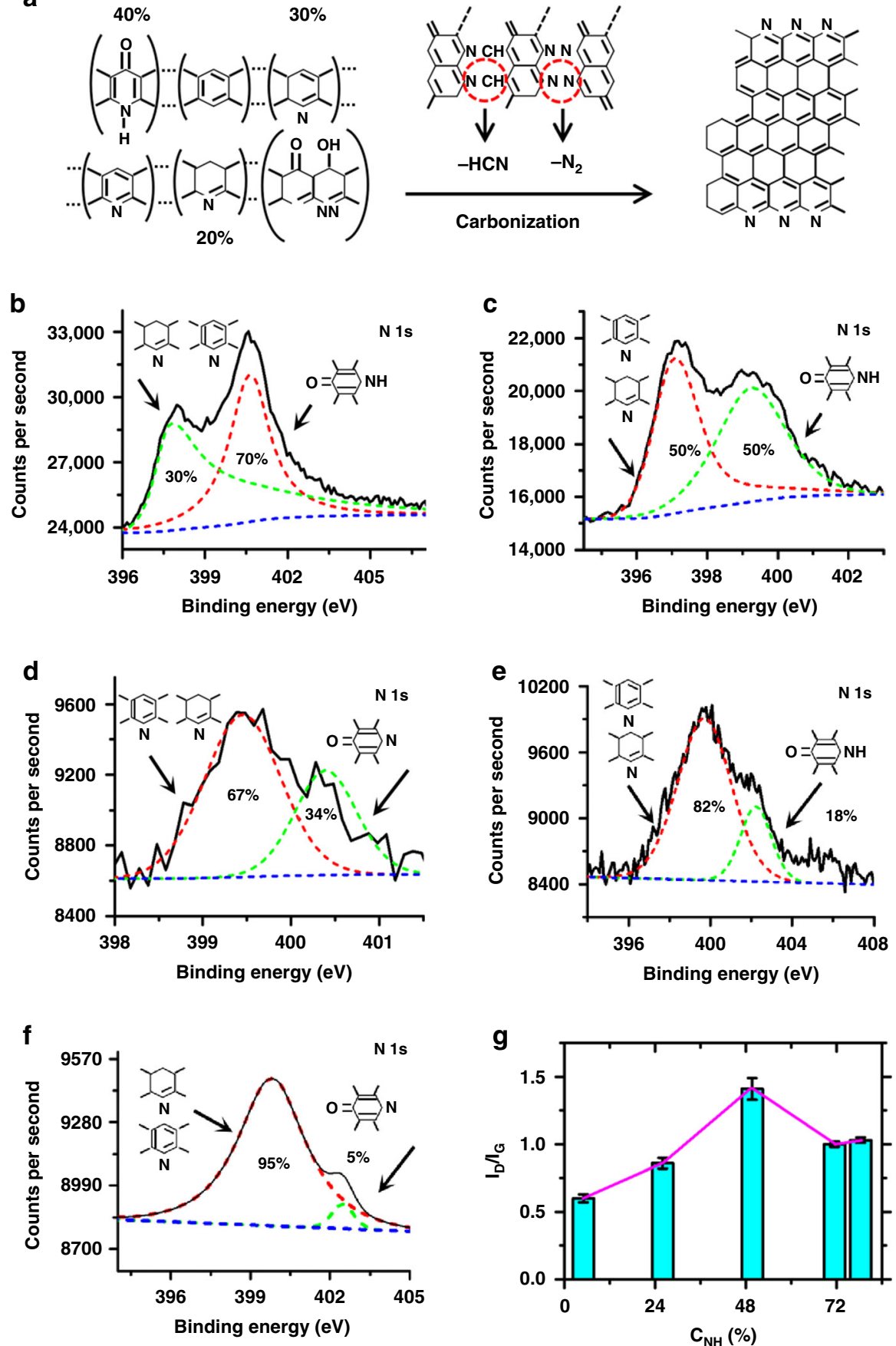

Fig. 3 Effect of the decyanation reaction on the crystalline structuring of wires. a Model reaction path from stabilized PAN to carbon. $\mathrm{N} 1 \mathrm{~s}$ X-ray photoelectron spectroscopy (XPS) spectra of $\mathbf{b}$ disordered carbon wires at $1200 \mathrm{~V}$ and of arrayed carbon nanowires at $\mathbf{c} 1200 \mathrm{~V}$, d $500 \mathrm{~V}$, and f $35 \mathrm{~V}$ with pretreatment of the silica surface in a nitrogen environment at $1000^{\circ} \mathrm{C}$. e N $1 \mathrm{~s} \mathrm{XPS} \mathrm{spectra} \mathrm{of} \mathrm{arrayed} \mathrm{carbon} \mathrm{nanowires} \mathrm{at} 35 \mathrm{~V}$ in the absence of nitrogen pretreatment of the silica surface. $\mathbf{g} / \mathrm{D} / l_{\mathrm{G}}$ as a function of the intensity of the acridine rings $\left(\mathrm{C}_{\mathrm{NH}}\right) ; n>5$ 
spectroscopy characterization was performed. The full $\mathrm{N}$ $1 \mathrm{~s}$ spectra presented in Fig. $3 \mathrm{~b}-\mathrm{f}$ demonstrate two typical peaks that are assigned to nitrogen atoms in the acridine ring and nitrogen atoms in the naphthyridine and hydronaphthyridine rings. For the porous microstructure of carbon wires with a diameter of $\sim 4.3 \mu \mathrm{m}$ (Fig. 2c), the corresponding $\mathrm{N} 1 \mathrm{~s}$ spectrum in Fig. 3c has two components, which are assigned to nitrogens in the acridine ring bonds (50\%) and in the naphthyridine and hydronaphthyridine rings $(50 \%)$. In comparison with that of carbon wires fabricated at $1200 \mathrm{~V}$ (Fig. 2a), the intensity of the acridine rings is weakened from $70 \%$ (Fig. $3 \mathrm{~b}$ ) to $50 \%$, revealing a more extensive decyanation reaction for the carbon wires in an array fabricated at $1200 \mathrm{~V}$. With copious decyanation, most of the reaction products that leave the carbonizing fiber are in the form of gases, including $\mathrm{HCN}, \mathrm{H}_{2} \mathrm{O}, \mathrm{O}_{2}, \mathrm{H}_{2}, \mathrm{CO}, \mathrm{NH}_{3}$, and $\mathrm{CH}_{4}{ }^{2}$, ultimately leading to a porous microstructure. The resulting intensity ratio of 1.41 (Fig. 2d) is much higher than the 0.96 in Fig. 2a but is comparable to the 1.38 from nanograin-based structuring, demonstrating that defects in the carbon wire reduce the degree of crystallization.

After the decrease in the nitrogen intensity of the acridine ring from 34 to $5 \%$ (Fig. $3 \mathrm{~d}, \mathrm{f}$ ), the resulting intensity ratio $\left(I_{\mathrm{D}} / I_{\mathrm{G}}\right)$ decreases from 0.78 to 0.64 (Fig. $2 \mathrm{~d}$ ), demonstrating the possibility of enhancing the decyanation reactions to improve the crystallinity. In the absence of nitrogen pretreatment of the silica surface, the resulting increase in the nitrogen intensity of the acridine ring from 5 to $18 \%$ (Fig. 3e, f) demonstrates that the presence of nitrogen at the surface facilitates more extensive decyanation reactions during carbonization, which leads to the improved crystallinity of carbon wire; this is similar to the results shown in Fig. 2a, b. Regardless of the porous defects in Fig. 2c, the effect of $I_{\mathrm{D}} / I_{\mathrm{G}}$ on the intensity of the acridine rings indeed reveals the improved crystallinity of the carbon wire with more extensive decyanation reactions, as shown in Fig. 3g.

The effect of nanowire diameter and decyanation on crystalline structuring reveals further insight into the underlying mechanism of graphitization at relatively low temperatures $\left(1000^{\circ} \mathrm{C}\right)$. Several previous studies have demonstrated that enhancing the degree of graphitization can, to a large extent, improve the physical and chemical nature of carbon nanowires ${ }^{14}$. Thus, the improvement in crystallinity with extensive decyanation reactions provides an attractive pathway for tailoring the effect of a crystalline surface structure on electrochemical properties.

\section{Electrochemical characterization using cyclic voltammetry}

In the case of very long experimental and theoretical time scales, hemispherical diffusion to microdisk electrodes $^{30-33}$ shows diffusion regimes depending on the CV scan rate or the EIS frequencies. Increasing the frequency to the characteristic value $\omega=D / d^{2}$, where $D$ is the diffusion coefficient of the analyte and $d$ is the distance between neighboring electrodes ${ }^{34,35}$, causes the transition from overlapping (Fig. 4a) to nonoverlapping (Fig. 4b) diffusion hemispheres. By further increasing the frequency to the characteristic value $\omega=D / a^{2}$, where $a$ is the radius of a single disc electrode, a planar diffusion regime is obtained; thus, a single electrode can be taken as a planar electrode. In the interval given by these two characteristic frequencies, the electrode array shows steady-state behavior with a sigmoidal cyclic voltammetry curve.

Cyclic voltammetry (CV) curves of the ferri/ferrocyanide $\left(\left[\mathrm{Fe}(\mathrm{CN})_{6}\right]^{3-/ 4-}\right)$ redox couple in aqueous solutions have often been used as a reference method to evaluate the electrochemical performance of a carbon electrode ${ }^{20,36,37}$. The CV curve from the disordered carbon wire mat in Fig. 2a shows macroelectrode behavior with anodic and cathodic peak currents in Fig. 4c, indicating partially overlapping diffusion hemispheres. Aligning the disordered carbon wires to a certain distance $(\sim 2.5 \mu \mathrm{m})$ at $1200 \mathrm{~V}$ results in a typical steady-state sigmoidal voltammogram with a steady-state current in Fig. 4d. This demonstrates the dependency of the nonoverlapping-tooverlapping diffusion regime on the distance of carbon wires in the characteristic frequency of $\omega=D / d^{2}$. By reducing the carbon wire diameter further from $\sim 4.3 \mu \mathrm{m}$ to $\sim 512 \mathrm{~nm}$, the interwire spacing further widens, leading to a decrease in the characteristic frequency $\left(\omega=D / d^{2}\right)$. The resulting CV curve in Fig. 4e still shows steady-state behavior but has a higher steady-state current compared to that of carbon wires arrayed at $1200 \mathrm{~V}$, which is likely derived from increasing the resistance of the carbon wire due to the decrease in diameter. Theoretical and experimental work ${ }^{30}$ on the diffusion of microelectrode arrays show that, in the case of a scan rate of $100 \mathrm{mV} / \mathrm{s}$ for $d \geq$ $100 a$, the dominating mode of diffusion is determined by the transition between the planar and hemispherical diffusion layers, leading to a sigmoidal shape in the $\mathrm{CV}$ curve. Clearly, the transition from the overlapping of the individual diffusion layers to planar diffusion layers over the entire electrode array in Fig. $4 \mathrm{f}$ is very distinct for a highly crystalline carbon nanowire array. The weakening of peak currents in the $\mathrm{CV}$ shows a monotonically increasing straight line (Fig. 4f), which is expected due to the large resistance in the thin carbon nanowires (Fig. 5d).

Lowering the applied voltage during NFES increases the specific surface area of carbon nanowires and subsequently improves the degree of graphitization, thus influencing the peak currents of carbon wires, as shown in Fig. 4h. The nanocrystalline-structured wires at $500 \mathrm{~V}$ clearly display at least a fourfold higher current than the porous-structured microwires at $1200 \mathrm{~V}$ and the highly 

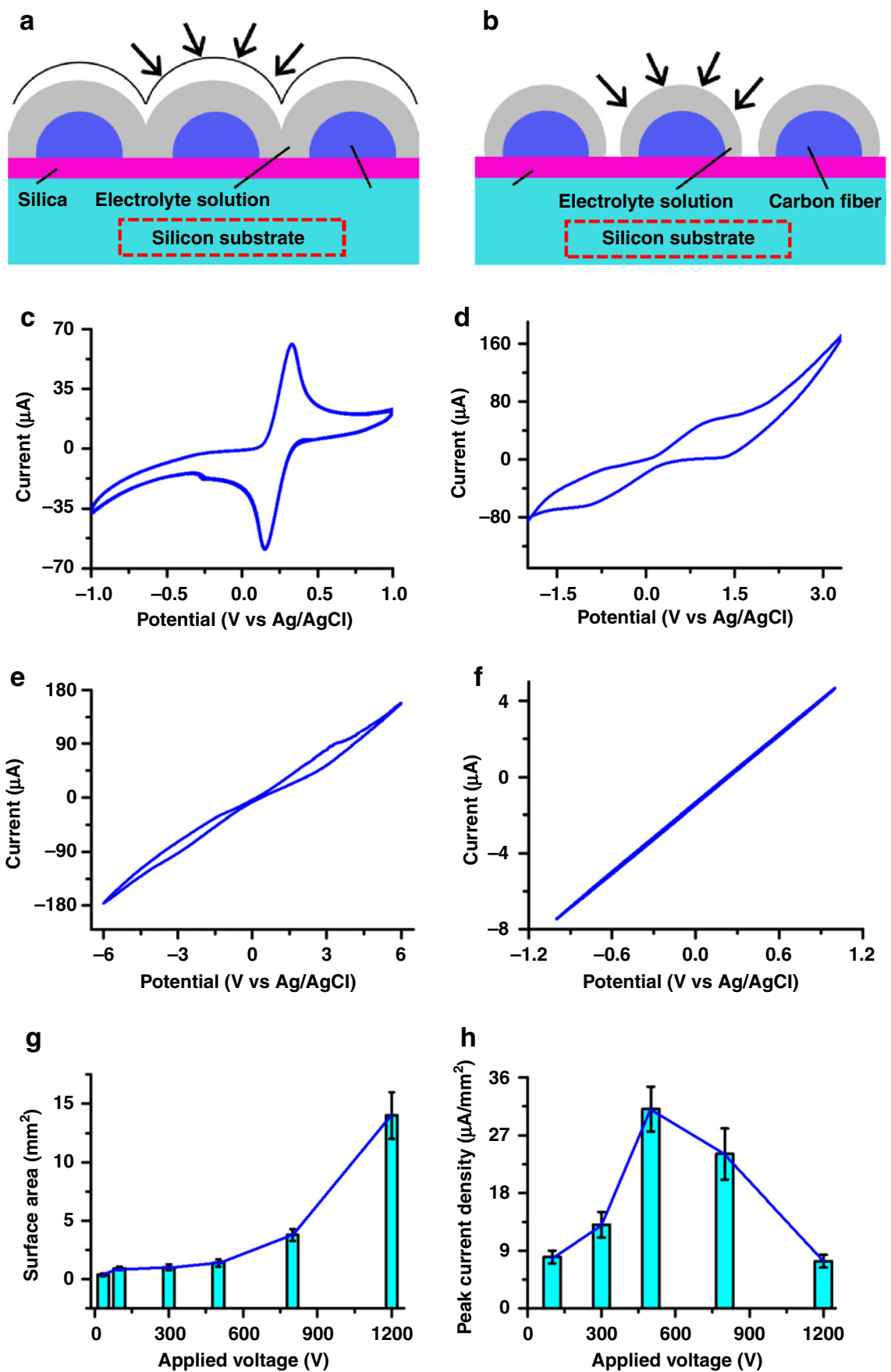

Fig. 4 Electrochemical characterization of crystalline carbon nanowire arrays based on cyclic voltammetry. Schematics showing the diffusion of carbon nanowires in the case of $\mathbf{a}$ overlapping and $\mathbf{b}$ nonoverlapping diffusion hemispheres. Cyclic voltammetry $(\mathrm{CV})$ curves of $5 \mathrm{mM} \mathrm{K} \mathrm{K}_{4} \mathrm{Fe}(\mathrm{CN})_{6} /$ $5 \mathrm{mM} \mathrm{K}_{3} \mathrm{Fe}(\mathrm{CN})_{6}$ in $0.1 \mathrm{M}$ phosphate buffer ( $\left.\mathrm{pH} 7.4\right)(1: 1$ mixture) for carbon wire mats disordered at c $1200 \mathrm{~V}$ and arrayed at d $1200 \mathrm{~V}$, e $800 \mathrm{~V}$, and $\mathbf{f} 35 \mathrm{~V}$, respectively; $n>8$. g Surface area and $\mathbf{h}$ peak current density of a carbon nanowire array as functions of applied voltage during NFES; $n>8$

crystalline nanowires at $35 \mathrm{~V}$. The higher surface area derived from the appearance of the nanocrystalline structure on the surface of the carbon nanowires is well correlated with the highest peak current of the carbon nanowire arrays at $500 \mathrm{~V}$. The highly crystalline carbon nanowire array at $100 \mathrm{~V}$ shows the smallest surface area in Fig. $4 \mathrm{~g}$ but has a peak current equivalent to that of the carbon nanowire array at $1200 \mathrm{~V}$ (Fig. $4 \mathrm{~h}$ ). This is most likely due to the highly graphitized structure increasing the electrical conductivity ${ }^{14}$. 


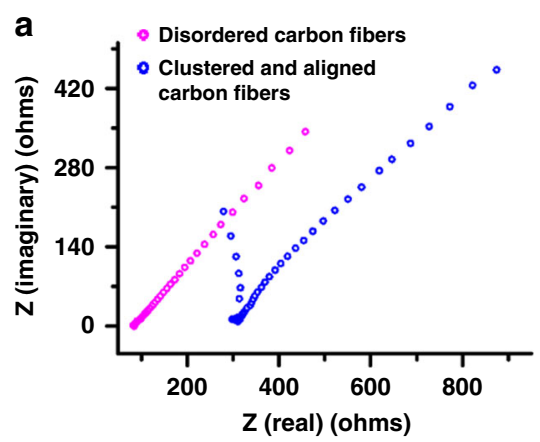

b
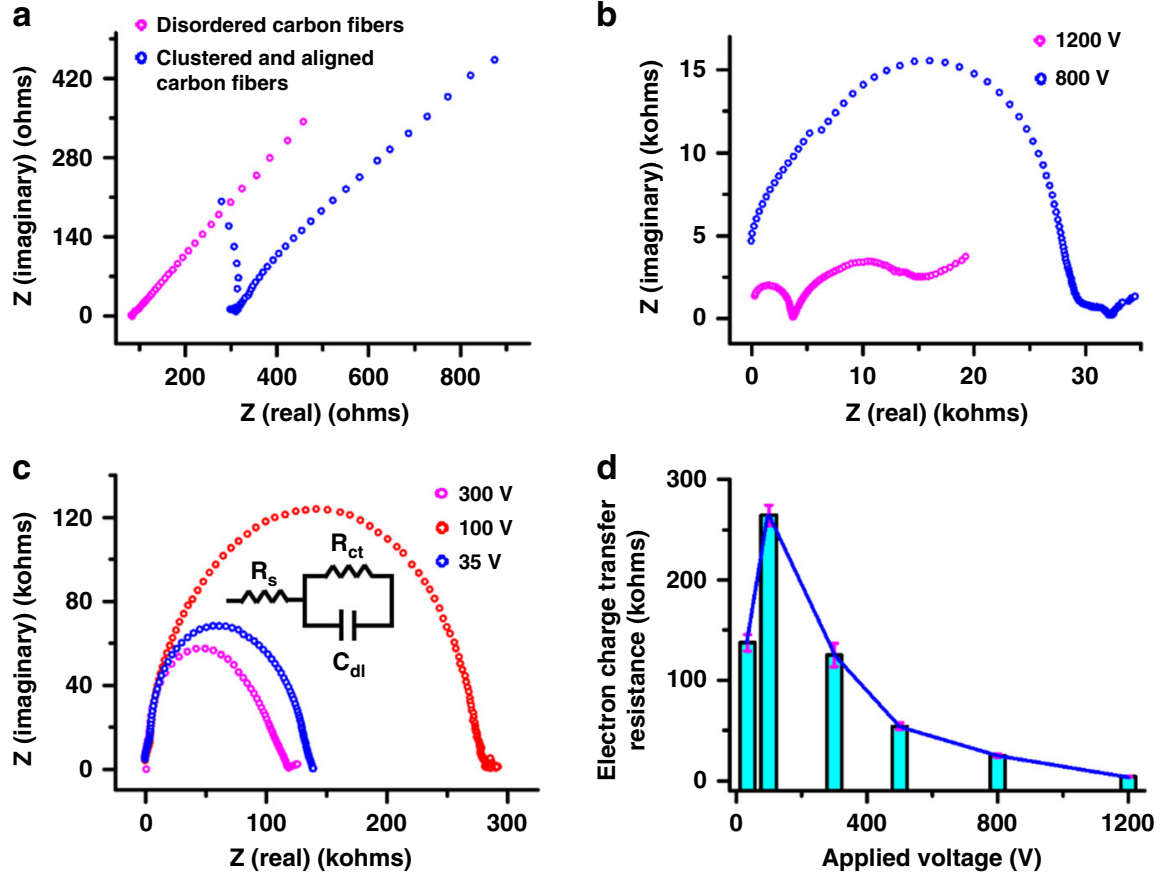

Fig. 5 Electrochemical characterization of crystalline carbon nanowire arrays based on impedance spectroscopy. Impedance spectra of the a disordered carbon mat at $1200 \mathrm{~V}$ and clustered and aligned carbon fiber mat at $1200 \mathrm{~V}$ along with the near-field carbon fiber arrays at $\mathbf{b} 1200$ and $800 \mathrm{~V}$ and $\mathbf{c}$ at 300,100 , and $35 \mathrm{~V} . R_{\mathrm{s}^{\prime}} R_{\mathrm{ct},}$ and $C_{\mathrm{d} \|}$ in $\mathbf{c}$ represent the solution resistance, electron transfer resistance, and double layer capacitance, respectively. AC amplitudes of $1000 \mathrm{mV}$ are applied to the carbon nanofibers in (a-c). Impedance spectra of near-field carbon fiber arrays at $35 \mathrm{~V}$ for AC amplitudes of 1000 and $25 \mathrm{mV} ; n>6$. d Electron charge transfer resistance as a function of the applied voltage during NFES; $n>10$

\section{Electrochemical characterization using impedance spectroscopy}

Electrochemical impedance spectroscopy (EIS) of the carbon nanowire array was performed using a solution of $5 \mathrm{mM} \mathrm{K} \mathrm{K}_{4} \mathrm{Fe}(\mathrm{CN})_{6} / 5 \mathrm{mM} \mathrm{K}_{3} \mathrm{Fe}(\mathrm{CN})_{6}$ in a $0.1 \mathrm{M}$ phosphate buffer ( $\mathrm{pH}$ 7.4). Impedance spectra were obtained at frequencies from $0.1 \mathrm{~Hz}$ to $1000 \mathrm{kHz}$ and at a $0 \mathrm{~V}$ dc potential vs. $\mathrm{Ag} / \mathrm{AgCl}$. The impedance spectra of the disordered carbon wire mat and clustered and aligned carbon wire mat are shown in Fig. 5a. Monotonically increasing straight lines with similar slopes at low frequencies are observed, reflecting a diffusion-limited process with the overlapping diffusion fields of all the participating wires. Upon the formation of the wire-to-wire distance derived from clustered and aligned carbon wires in Fig. 2a, the impedance spectroscopy in Fig. 5a shows a quarter circle at high frequencies and a straight line at low frequencies. This is due to the weakening of the diffusion gradients $(f)$ according to Eq. (3): ${ }^{34}$

$$
f=\frac{4 \tilde{\alpha}}{\pi d^{2}}
$$

where $f$ is the diffusion gradient in front of each individual -ultramicroelectrode and $\hat{\alpha}$ denotes the arithmetical average of the oxidation and reduction diffusion coefficients of $\left[\mathrm{Fe}(\mathrm{CN})_{6}\right]^{3-/ 4-}$.
By arraying all the carbon nanowires and maintaining a wire-to-wire spacing of $\sim 2.5 \mu \mathrm{m}$, the impedance spectrum shows two semicircles connected together at high frequencies and a relatively short straight line with a similar slope (diffusion limitation) at the lowest frequencies, as shown in Fig. 5b. Theoretical and experimental work ${ }^{34}$ on the impedance of ultramicroelectrode arrays exhibit a semicircle at low frequencies in addition to the wellknown semicircle at higher frequencies, demonstrating overlap of the diffusion gradients. The change from overlapping diffusion to diffusion limitation is shown for carbon nanowire arrays at $1200 \mathrm{~V}$. This demonstrates the weakening of the overlap of the diffusion gradients compared to that of the clustered and aligned carbon wires.

By reducing the applied voltage from 1200 to $800 \mathrm{~V}$, which is accompanied by a decrease in the wire diameter from $\sim 4.3 \mu \mathrm{m}$ to $\sim 512 \mathrm{~nm}$, the impedance spectrum changes to a large semicircle with a small straight line, as shown in Fig. 5b. The semicircle diameter at higher frequencies is governed by the charge transfer resistance $\left(R_{\text {et }}\right)$ related to the electron transfer rate of the redox species on the electrode surface. The region at low frequencies again represents a line with a similar slope, showing mass transfer control or a diffusion limitation. Previously, in the case of three-dimensional hemispherical diffusion to an array of microdisk electrodes ${ }^{31-34}$, the 
impedance spectrum showed a semicircle for frequencies between the characteristic frequencies of $\omega=D / d^{2}$ and $\omega=D / a^{2}$. The transition from overlapping diffusion to three-dimensional hemispherical diffusion at high frequencies is shown to observe the changes between the impedance spectra of carbon nanowire arrays at 1200 and at $800 \mathrm{~V}$. The impedance spectrum of the carbon nanowire array at $800 \mathrm{~V}$ is expected due to the lower density of arrayed carbon wire ${ }^{20}$, which is derived from widening the wire-to-wire distance with decreasing wire diameter.

Reducing the diameter of the arrayed carbon wire from $\sim 512$ to $\sim 40 \mathrm{~nm}$ with decreasing applied voltage from 800 to $35 \mathrm{~V}$ results in changes in the impedance spectrum from a large semicircle with a small straight line to just a single semicircle without a Warburg impedance element (Fig. 5c). This case allows for the process from the overlapping diffusion of all the participating wires to the complete reaction kinetics determined by electron transfer. Theoretical work ${ }^{35}$ has shown that a smaller electrode radius leads to a larger electrode admittance per unit area, which allows the susceptance at low frequencies to remain negligible. In addition, decreasing the nanowire diameter increases the lower frequency limit at which the susceptance (imaginary part of the impedance) becomes lower than the conductance (real part of the impedance). Thus, the impedance spectrum of the highly crystalline carbon nanowire array at $35 \mathrm{~V}$ shows a semicircle for $\omega=D / a^{2}$ " $D / d^{2}$. These results demonstrate the ability to control the diffusion regimes by altering the wire spacing and nanowire size.

In most studies, the semicircle spectrum from carbon nanowire arrays is meaningful only when ac signals with amplitudes $\leq 25 \mathrm{mV}$ are applied ${ }^{38,39}$. By reducing the amplitude from $1000 \mathrm{mV}$ to $25 \mathrm{mV}$, the impedance spectrum of the highly crystalline carbon nanowires in Fig. 5c still shows a single semicircle without scattered data points, demonstrating the high signal-to-noise ratio compared to that of typical carbon nanowire electrode arrays $^{20}$. Therefore, highly crystalline carbon nanowire arrays can be selected as potential candidates for DNA switching ${ }^{40}$.

The impedance spectra were fitted by means of an equivalent Randles circuit with capacitance, charge transfer resistance, solution resistance, and Warburg elements. The charge transfer resistance related to the overall surface area of the carbon nanowire arrays corresponds to the diameter of the semicircle in the highfrequency domain of the impedance spectra. Figure $5 \mathrm{~d}$ shows the increase in charge transfer resistance as the applied voltage is decreased from 1200 to $100 \mathrm{~V}$; notably, this is accompanied by a decrease in the wire diameter from $\sim 4.3 \mu \mathrm{m}$ to $\sim 120 \mathrm{~nm}$ and the microstructural transformation from a porous microstructure to a nanocrystalline microstructure. It is well known from theoretical and experimental work on carbon microelectrodes that the smaller the electrode surface area is, the greater the charge transfer resistance ${ }^{41}$. The monotonic correlation between the charge transfer resistance and wire diameter in Fig. $5 \mathrm{~d}$ is expected in accordance with the results of carbon microelectrodes. Clearly, by changing the wire diameter in the carbon nanowire arrays, the semicircle scale at high frequencies can be adjusted.

A previous study ${ }^{14}$ on the correlation between microstructures and properties showed that enhancing graphitic microstructures could improve the bulk characteristics of carbon fabrics, resulting in an increase in conductivity from $\sim 200 \mathrm{~S} / \mathrm{m}$ to $\sim 5000 \mathrm{~S} / \mathrm{m}$ with improving graphitization. The charge transfer resistance of highly crystalline carbon nanowire arrays at $35 \mathrm{~V}$ is unusually low and is half of that of carbon nanowire arrays at $100 \mathrm{~V}$ (Fig. 5d). This is most likely due to the further improvement of the graphitic microstructure as the voltage is decreased from 100 to $35 \mathrm{~V}$ (Fig. 2d). Thus, the combination of these factors, including the wire-to-wire spacing, nanoscale diameter, and graphitic microstructure, leads to a distinct semicircle of highly crystalline carbon nanowire arrays in EIS.

\section{Conclusion}

In the state-of-the-art fabrication of carbon nanowires with the carbon-nano-electro-mechanical system approach, the growth of a graphitic microstructure is typically limited to the use of a Ni catalyst. In this work, we demonstrated a novel catalyst-free fabrication process for arrays of highly graphitized carbon nanowires with various surface nanostructures. It was developed by minimizing the polymer fiber diameter with ultralowvoltage NFES. The linear speed of the spinneret and the rotational speed of the collector allowed for better control of the fiber-to-fiber distance in the fiber arrays. The stabilization at $115^{\circ} \mathrm{C}$ and subsequent carbonization converted these polymer nanofibers arrayed on a silica surface into carbon nanowires. The thickness control of carbon nanowires was conveniently adjusted by tuning the applied voltage during NFES. Aligning the wires on a nitrogen-pretreated silica surface allowed for stress decyanation to improve the graphitic content of the carbon nanowires. The presented diameter control showed the ability to transform a porous-microstructured carbon wire into a highly crystalline nanostructure via nanocrystalline structuring.

Various electrochemical behaviors were observed in the obtained CV curves when reducing the wire diameter because that altered the structure on the surface of the carbon wire and improved the graphitic structure. The typical CV curve showing macroelectrode behavior with anodic and cathodic peak currents was altered to a sigmoidal CV curve with the steady-state current dominated 
by radial diffusion. This is a characteristic behavior observed for carbon nanowire arrays where there is no overlap of the diffusion hemispheres from neighboring electrodes. Compared to three-dimensional carbon nanowire electrode arrays with wire diameters of $\sim 100 \mathrm{~nm}$, two-dimensional carbon nanowire arrays with diameters of $\sim 512 \mathrm{~nm}$ easily obtained low steady-state currents during cyclic voltammetry, which has been regarded as a potential advantage for biosensing applications. -The nanocrystalline structure on the surface of graphitized carbon wires rendered extremely high peak currents in their CV curve. More importantly, highly crystalline carbon nanowire arrays showed a linear $\mathrm{CV}$ curve without anodic and cathodic peak currents, representing the special characteristics of the highly graphitized structures in the nanoscale carbon wires. The charge transfer resistance could be controlled by varying the diameter of the carbon nanowires. In the EIS spectra of the highly crystalline carbon nanowire arrays, the abnormal decrease in the charge transfer resistance confirmed the influence of the graphitized structure on the electrochemical behavior.

The electrochemical performance of the crystalline carbon nanowire arrays with various surface nanostructures makes them potential candidates for biochemical sensors with lower detection limits and as devices for electrochemical energy storage. Graphitized carbon submicrowires with a nanocrystalline structure could allow for amplified biosensing via redox cycling and enhanced capacitive energy storage in microsupercapacitors. The linear CV curve of highly crystalline carbon nanowires shows the ability to permit stable data collection for ultrasensitive biological detection. In particular, a further increase in the surface area of -nanocrystalline-structured carbon wires could allow for high-power supercapacitors.

\section{Materials and methods}

The electrospinning solution (9\% PAN) was prepared by dissolving PAN (150000 mw, Sigma Aldrich, St. Louis, $\mathrm{MO}$ ) in DMF. Using vortex mixing at $30 \mathrm{RPM}$, mixtures of PAN/DMF were allowed to freely diffuse at different temperatures. For the NFES experiments, we used a $3 \mathrm{~mL}$ syringe mounted on a syringe pump to dispense the highest conductivity ink at a feed rate below $10 \mathrm{~nL} / \mathrm{min}$. Silicon substrates coated with silicon dioxide were mounted in the grooves and convex areas on the drum with carbon tape. The NFES voltage was applied between the dispensing needle and the grounded drum. It should be noted that regular arrays were achieved as long as $\omega \geq$ $400 \mathrm{RPM}$ and $v \geq 80 \mu \mathrm{m} / \mathrm{s}$ were achieved at the same time. Fabrication of carbon nanowire arrays derived from PAN nanofiber arrays consisted of oxidization in the air at $115^{\circ} \mathrm{C}$ (named stabilization) and subsequent carbonization in a furnace with an inert nitrogen atmosphere at $1000^{\circ} \mathrm{C}$ (heating ramp rate of $15^{\circ} \mathrm{C} / \mathrm{min}$ ) (Fig. $1 \mathrm{~b}$ ).
In general, the graphitic nature of PAN-derived carbons was evaluated based on Raman spectroscopy, a standard nondestructive analysis tool. The Raman spectrometer with a DXR microscope (Thermo Fisher) and equipped with a $532 \mathrm{~nm}$ excitation laser assessed the corresponding carbon wire arrays. By moving the platform on which the sample was located, the wire was moved to a location just below the marked laser spot. Linear scanning was implemented with a step size of 100 nanometers. For each step, data were collected by exciting the laser, ultimately resulting in obtaining the Raman spectrum of a carbon nanowire. We used the lens for an average of approximately 20 times per sample. To correlate the influence of denitrogenation and the decyanation reaction on the graphitic structure, X-ray photoelectron spectroscopy was performed.

Atomic force microscopy (AFM) (Bruker Dimension Icon) and scanning electron microscopy (SU8220) allowed for a detailed study of the surface nanostructures in the resulting carbon fibers. The topology of carbon fibers was studied with the tapping mode to investigate the influence of the applied voltage on various surface nanostructures.

A traditional three-electrode configuration consisting of an $\mathrm{Ag} / \mathrm{AgCl}$ reference electrode, Pt counter electrode, and carbon working electrode was used. To probe the carbon/ electrolyte interface, a GPSTAT12 potentiostat/galvanostat equipped with a frequency response analyzer module was employed. Cyclic voltammetry $(\mathrm{CV})$ of the ferri/ferrocyanide $\left(\left[\mathrm{Fe}(\mathrm{CN})_{6}\right]^{3-/ 4-}\right)$ redox couple in aqueous solutions have often been used as a reference method to evaluate the electrochemical performance of a carbon electrode $^{20,36,37}$.

Electrochemical impedance spectroscopy (EIS) of the carbon nanofiber array was performed using a solution containing $5 \mathrm{mM} \mathrm{K}_{4} \mathrm{Fe}(\mathrm{CN})_{6} / 5 \mathrm{mM} \mathrm{K}_{3} \mathrm{Fe}(\mathrm{CN})_{6}$ in a $0.1 \mathrm{M}$ phosphate buffer ( $\mathrm{pH}$ 7.4). Impedance spectra were taken at frequencies from $0.1 \mathrm{~Hz}$ to $1000 \mathrm{kHz}$ and at a dc potential of $0 \mathrm{~V}$ vs. $\mathrm{Ag} / \mathrm{AgCl}$. The number of experiments $(n)$ is indicated in the figure legends.

\section{Acknowledgements \\ This research was supported by the National Key R\&D Program of China (2020YFB2009002) and the National Natural Science Foundation of China (51875084). The support provided by the China Scholarship Council (CSC) during the visit of Jufeng Deng to the University of California, Irvine is acknowledged.}

\section{Author details}

${ }^{1}$ School of Mechanical Engineering, Dalian University of Technology, Dalian 116023, China. ${ }^{2}$ Mechanical and Aerospace Engineering, University of California, Irvine, CA 92617, USA. ${ }^{3}$ Chemical and Biomolecular Engineering, University of California, Irvine, CA 92517, USA. ${ }^{4}$ School of Engineering and Science, Tecnologico de Monterrey, Monterrey, NM 64849, Mexico

\section{Author contributions}

$J \mathrm{D}, \mathrm{CL}, \mathrm{DS}$, and MM conceived the project. JD, CL, DS, and MM developed the process for growing carbon nanowires. JD, CL, DS, and MM interpreted the 
data and developed the figures. JD, CL, DS, and MM wrote the manuscript. JD transferred and prepared the AFM samples. JD performed the AFM characterization and data analysis. JD performed the AFM, XPS, CV, and EIS characterization tests and the data analysis. JD, CL, DS, and MM edited the manuscript. All authors discussed the data and contributed to the final manuscript.

\section{Conflict of interest}

The authors declare no competing interests.

Received: 29 July 2021 Revised: 25 November 2021

Accepted: 3 December 2021

Published online: 04 February 2022

\section{References}

1. Jeffries, R. Prospects for carbon fibers. Nature 232, 304-307 (1971).

2. Frank, E. et al. Carbon fibers: precursor systems, processing, structure, and properties. Angew. Chem. Int. Ed. 53, 5262-5298 (2014).

3. Ra, E. J., Raymundo-Pinero, E., Lee, Y. H. \& Beguin, F. High power supercapacitors using polyacrylonitrile-based carbon nanofiber paper. Carbon 47, 2984-2992 (2009).

4. Huang, J., Liu, Y. \& You, T. Carbon nanofiber based electrochemical biosensors: a review. Anal. Methods 2, 202-211 (2010).

5. Mitchell, R. R., Gallant, B. M., Thompson, C. V. \& Shao-Horn, Y. All - carbon nanofiber electrodes for high - energy rechargeable $\mathrm{Li}-\mathrm{O}_{2}$ batteries. Energy Environ. Sci. 4, 2952-2958 (2011).

6. Bacon, R. Growth, structure, and properties of graphite whiskers. J. Appl. Phys. 31, 283-290 (1960).

7. Balandin, A. A. Thermal properties of graphene and nanostructured carbon materials. Nat. Mater. 10, 569-581 (2011).

8. Lee, C., Wei, X., Kysar, J. W. \& Home, J. Measurement of the elastic properties and intrinsic strength of monolayer graphene. Science 321, 385-388 (2008).

9. Xu, Z. \& Gao, C. Graphene fiber: a new trend in carbon fibers. Mater. Today $\mathbf{1 8}$ 480-492 (2015).

10. Zamora-Ledezma, C. et al. Liquid crystallinity and dimensions of surfactantstabilized sheets of reduced graphene oxide. J. Phys. Chem. Lett. 3, 2425-2430 (2012).

11. Kou, L. et al. Coaxial wet-spun yarn supercapacitors for high-energy density and safe wearable electronics. Nat. Commun. 5, 3754 (2014).

12. Yang, Z. et al. Photovoltaic wire derived from a graphene composite fiber achieving an 8.45\% energy conversion efficiency. Angew. Chem. 125, 7693-7696 (2013).

13. Xin, G. et al. Highly thermally conductive and mechanically strong graphene fibers. Science 349, 1083-1087 (2015).

14. Ghazinejad, M. et al. Graphitizing non-graphitizable carbons by stress-induced routes. Sci. Rep. 7, 16551 (2017)

15. Boskovic, B. O. et al. Large-area synthesis of carbon nanofibers at room temperature. Nat. Mater. 1, 165-168 (2002).

16. Deng, J., Liu, C. \& Madou, M. Ultra-thin carbon nanofibers based on graphitization of near-field electrospun polyacrylonitrile. Nanoscale 12, 10521-10531 (2020).

17. George, D. et al. Fabrication of patterned graphitized carbon wires using low voltage near-field electrospinning, pyrolysis, electrodeposition, and chemical vapor deposition. Microsyst. Nanoeng. 6, 7 (2020).

18. Morad, M. R., Rajabi, A., Razavi, M. \& Pejman, S. R. Sereshkeh, a very stable high throughput Taylor cone-jet in electrohydrodynamics. Sci. Rep. 6, 38509 (2016).
19. Dotivala, A. C., Puthuveetil, K. P. \& Tang, C. Shear force fiber spinning: process parameter and polymer solution property considerations. Polymer 11, 294 (2019).

20. Siddiqui, S., Arumugam, P. U., Chen, H., Li, J. \& Meyyappan, M. Characterization of carbon nanofiber electrode arrays using electrochemical impedance spectroscopy: effect of scaling down electrode size. ACS Nano 4, 955-961 (2010).

21. Liu, J. et al. Study on the oxidative stabilization of polyacrylonitrile fibers by microwave heating. Polym. Degrad. Stab. 150, 86-91 (2018).

22. Khayyam, H. et al. PAN precursor fabrication, applications and thermal stabilization process in carbon fiber production: experimental and mathematical modelling. Prog. Mater. Sci. 107, 100575 (2020).

23. Zhao, R.-X. et al. Influence of heating procedures on the surface structure of stabilized polyacrylonitrile fibers. Appl. Surf. Sci. 433, 321-328 (2018).

24. Inagaki, M., Yang, Y. \& Kang, F. Carbon nanofibers prepared via electrospinning. Adv. Mater. 24, 2547-2566 (2012).

25. Ferrer-Argemi, L. et al. Size - dependent electrical and thermal conductivities of electro - mechanically - spun glassy carbon wires. Carbon 130, 87-93 (2018).

26. Johnson, J. W., Marjoram, J. R. \& Rose, P. G. Stress graphitization of polyacrylonitrile based on carbon fiber. Nature 221, 357-358 (1969).

27. Sharma, S., Sharma, A., Cho, Y.-K. \& Madou, M. Increased graphitization in electrospun single suspended carbon nanowires integrated with carbonMEMS and carbon-MEMS platforms. ACS Appl. Mater. Interfaces 4, 34-39 (2012).

28. Venugopal, G., Jung, M., Suemitsu, M. \& Kim, S. Fabrication of nanoscale threedimensional graphite stacked junctions by focused-ion-beam and observation of anomalous transport characteristics. Carbon 49, 2766-2772 (2011).

29. Zussman, E. et al. Mechanical and structural characterization of electrospun PAN- derived carbon nanofibers. Carbon 43, 2175-2185 (2005).

30. Guo, J. \& Lindner, E. Cyclic voltammograms at coplanar and shallow recessed microdisk electrode arrays: guidelines for design and experiment. Anal. Chem. 81, 130-138 (2009).

31. Fleischmann, M., Pons, S. \& Daschbach, J. The AC impedance of spherical, cylindrical, disk and ring microelectrodes. J. Electroanal. Chem. 17, 1-26 (1991).

32. Fleischmann, M. \& Pons, S. The behavior of microdisk and microring elec trodes. Mass transport to the disk in the Unsteady State: The AC Response. J. Electroanal. Chem. 250, 277-283 (1988).

33. Abrantes, L. M., Fleischmann, M., Peter, L. M., Pons, S. \& Scharifker, B. R. On the diffusional impedance of microdisc electrodes. J. Electroanal. Chem. 256, 229-233 (1988).

34. Koster, O., Schuhmann, W., Vogt, H. \& Mokwa, W. Quality control of ultramicroelectrode arrays using cyclic voltammetry, electrochemical impedance spectroscopy and scanning electrochemical microscopy. Sens. Actuators B 76 573-581 (2001).

35. Hepel, T. \& Osteryoung, J. Electrochemical characterization of electrodes with submicrometer dimensions. J. Electrochem. Soc. 133, 757 (1986).

36. Hees, J. et al. Nanocrystalline diamond nanoelectrode arrays and ensembles. ACS Nano 5, 3339-3346 (2011).

37. McCreery, R. L. Advanced carbon electrode materials for molecular electrochemistry. Chem. Rev. 108, 2646-2687 (2008).

38. Barbero, G., Alexe-lonescu, A. L. \& Lelidis, I. Significance of small voltage in impedance spectroscopy measurements on electrolytic cells. J. Appl. Phys. 98 1137031-1137035 (2005).

39. Freire, F. M., Barbero, G. \& Scalerandi, M. Electrical impedance for an electrolytic cell. Phys. Rev. E. 73, 051202-051211 (2006).

40. Rant, U. et al. Detection and size analysis of proteins with switchable DNA layers. Nano Lett. 9, 1290-1295 (2009).

41. Mantis, I. et al. Suspended highly 3D interdigitated carbon microelectrodes. Carbon 179, 579-589 (2021) 